\title{
Reply to \\ "Comment on The COVID-19 infodemic does not affect vaccine acceptance"
}

\author{
Carlo M. Valensise ${ }^{1}$, Matteo Cinelli ${ }^{2,3}$, Matthieu Nadini ${ }^{4,6}$, \\ Alessandro Galeazzi ${ }^{5}$, Antonio Peruzzi ${ }^{2}$, Gabriele Etta ${ }^{7}$, Fabiana \\ Zollo $^{2}$, Andrea Baronchelli, ${ }^{4, *}$, and Walter Quattrociocchi ${ }^{7, *}$ \\ ${ }^{1}$ Enrico Fermi Research Center, Piazza del Viminale, 1 - 00184 Roma (IT) \\ ${ }^{2} \mathrm{Ca}$ ' Foscari, University of Venice - Department of Environmental Sciences, \\ Informatics and Statistics, Via Torino 155, 30172 Venezia (IT) \\ ${ }^{3}$ Italian National Research Council - Institute for Complex Systems Via dei Taurini \\ 19, 00185 Roma (IT) \\ ${ }^{4}$ City University of London, Department of Mathematics, London EC1V 0HB, UK \\ ${ }^{5}$ University of Brescia, Via Branze, $59-25123$ Brescia (IT) \\ ${ }^{6}$ The Alan Turing Institute, British Library, 96 Euston Road, London NW12DB, UK \\ ${ }^{7}$ Sapienza University of Rome — Department of Computer Science, Viale Regina \\ Elena, 295 - 00161 Roma (IT) \\ *Corresponding authors: abaronchelli@turing.ac.uk, \\ walter.quattrociocchi@uniroma1.it
}

Our recent working paper [1] shows that the announcement of, and news about, the COVID-19 vaccines triggered huge interest on social media in six European countries, affecting attention patterns and the modality in which information was spreading (e.g., links vs photos). It then analyses answers about vaccination intention from the COVID-19 Symptom Survey [2], part of the broader Facebook Data for Good project, collected between 23 January and 30 April 2021, and found a substantially flat behaviour (average slope is compatible with zero according to t-test). Even during the days following the dramatic EMA suspension of the AstraZeneca vaccine, the survey shows only a weak and temporary drop in vaccine intention. Finally, the appendix of the paper shows that the same survey conducted over other countries reveal a smooth not necessarily flat - behaviour. We conclude that this might be a case in which the overall online and offline signals are uncorrelated, and suggest that more research is needed to understand the relationship between online and offline behaviour.

Gallotti et al [3] raised concerns that our analysis might be missing other signals of correlation, and replicated a similar study on more countries using a less fine grained survey to find that "a broad spectrum of patterns includ- 
ing both positive and negative correlations [between online signal and survey responses] as well as no correlations at all". We think that these observations are helpful in stimulating a broader discussion, and our working paper [1] offers valid arguments that address the concerns. While successive versions of our paper will highlight this even more clearly, we detail below the main points who might be of interest for the reader of Gallotti et al.'s comment.

First, Gallotti et al. raise interesting "conceptual" points centered on the literature on the relationship between social media and effects on vaccine hesitancy. While we are well-aware that individual online behaviour can be a strong predictor of (intention of) offline behaviour and exposure to new online (mis)information may alter the latter, these points are not incompatible with the findings of our paper. As briefly mentioned in the abstract and conclusions, in fact, the lack of evidence for a correlation between online conversation and overall COVID-19 vaccine intention is compatible with a scenario where users seek for, and interact with, online information that agrees with their current beliefs or, as we wrote, our result is "consistent with the concept of echo chambers enhancing conservatism of opinions". Then, Gallotti et al. raise a number of more technical points. We do not believe they weaken our conclusions. We address them one by one. (1) Infodemic definition. As detailed in our paper, we adopted the WHO definition describing infodemic as "overabundance of information - some accurate and some not - that occurs during an epidemic" ${ }^{1}$. We welcome other studies using different definitions (for a recent review see [4]). (2) Twitter Dataset. The authors of [3] highlight that our Twitter database lacks deleted tweets. Hence, we may have underestimated the intensity of the infodemic. A stronger infodemic would strengthen the main finding of the paper that a 'strong' infodemic is mirrored by a substantially flat vaccine acceptance. Furthermore, we mainly focused on Facebook users and their content consumption, since they are part of the same population of the surveys. Twitter was mainly used to further support the change of information consumption patterns. Moreover, the Twitter dataset investigated in [3] only partially overlaps with ours, as the keywords used for posts retrieval are different. (3) Vaccine survey. The Facebook survey [2] has an exceptionally high number of daily respondents. Such a granularity was required to perform the analysis of the EMA event and, to the best of our knowledge, no comparable surveys are publicly available. We welcome studies analysing other datasets. (4) Absence of correlation. Gallotti et al. perform a correlation analysis between infodemic indicators obtained from the COVID-19 Infodemic Observatory ${ }^{2}$ and the percentage of respondents who answered negatively to the question about vaccine acceptance in MIT COVID-19 Survey ${ }^{3}$. They find that the two signals correlate for some countries, anti-correlate for others, and show no correlations for a third group a signal difficult to interpret that would require further investigation. Furthermore, not only the survey used in [3] has only 19 data points over 9 months, but the time frame analysed in the comment is only partially overlapping with

\footnotetext{
${ }^{1}$ https://www.who.int/teams/risk-communication/infodemic-management

${ }^{2}$ https://covid19obs.fbk.eu/\#/

${ }^{3}$ https://covidsurvey.mit.edu
} 
the one investigated in our working paper (from July 6, 2020 to March 28, 2021 and from January 23, 2021 and April 30, 2021, respectively). Specifically, the MIT COVID-19 Survey provides only two data points in the overlap between the two time windows. (5) Time scales. The comment [3] stresses that building cognitive frames and vaccine hesitancy are slow processes. Hence, the analysis of short time periods might be unable to capture long term effects. We note that COVID-19 vaccines were announced in late 2020 and by mid 2021 the vaccination campaign was mature in the considered countries, hence short time effects (or lack thereof) are crucial in this case. We would certainly welcome future studies addressing the long term aspects of our study.

Finally, an overarching point in [3] is that infodemic, vaccine hesitancy, and their interplay are complex phenomena, and that they should be treated as such. We agree with both points. It is the duty of researchers to publish their results as they become available, and to offer them to the scrutiny of their peers. Our study has no pretence of closing the debate. On the contrary, it aims to stimulate further research and debate on such a topical issue. We are

pleased to acknowledge it has succeeded so soon and we hope it will spark more investigations in the future.

\section{References}

[1] Carlo M Valensise, Matteo Cinelli, Matthieu Nadini, Alessandro Galeazzi, Antonio Peruzzi, Gabriele Etta, Fabiana Zollo, Andrea Baronchelli, and Walter Quattrociocchi. The covid-19 infodemic does not affect vaccine acceptance. arXiv preprint arXiv:210\%.07946v1, 2021.

[2] Junchuan Fan, Yao Li, Kathleen Stewart, Anil R. Kommareddy, Andres Garcia, Jinyi Ma, Zheng Liu, Joe O'Brien, Adrianne Bradford, Xiaoyi Deng, Samantha Chiu, Frauke Kreuter, Neta Barkay, Alyssa Bilinski, Brian Kim, Tal Galili, Daniel Haimovich, Sarah LaRocca, Stanley Presser, Katherine Morris, Joshua A Salomon, Elizabeth A. Stuart, Ryan Tibshirani, Tali Alterman Barash, Curtiss Cobb, Andi Gros, Ahmed Isa, Alex Kaess, Faisal Karim, Roee Eliat, Ofir Eretz Kedosha, Shelly Matskel, Roee Melamed, Amey Patankar, Irit Rutenberg, Tal Salmona, and David Vannette. COVID19 world symptom survey data API., 2020. Carnegie Mellon University, Pennsylvania, United States.

[3] Riccardo Gallotti, Federico Pilati, Pier L Sacco, and Manlio De Domenico. Comment on "the covid-19 infodemic does not affect vaccine acceptance", Jul 2021.

[4] Felix M Simon and Chico Q Camargo. Autopsy of a metaphor: The origins, use and blind spots of the 'infodemic'. New Media 8 Society, 0(0):14614448211031908, 0 . 